

\title{
Effect of Ta buffer layer and thickness on the structural and magnetic properties of Co thin films
}

K. Vahaplar, S. Tari, H. Tokuc, and S. Okur

Citation: Journal of Vacuum Science \& Technology B 27, 2112 (2009); doi: 10.1116/1.3196784

View online: http://dx.doi.org/10.1116/1.3196784

View Table of Contents: http://scitation.aip.org/content/avs/journal/jvstb/27/5?ver=pdfcov

Published by the AVS: Science \& Technology of Materials, Interfaces, and Processing

\section{Articles you may be interested in}

Effect of buffer layer and external stress on magnetic properties of flexible FeGa films

J. Appl. Phys. 113, 17A901 (2013); 10.1063/1.4793602

Influence of a TiO2 buffer layer on the magnetic properties of anatase $\mathrm{Co}: \mathrm{TiO} 2$ thin films

J. Appl. Phys. 111, 083917 (2012); 10.1063/1.4706570

Morphological and magnetic properties of Co nanoparticle thin films grown on Si $3 \mathrm{~N} 4$

J. Appl. Phys. 102, 053901 (2007); 10.1063/1.2775172

Effect of annealing on magnetotransport and structural properties of Co/Cu multilayers with Ta buffer layer J. Appl. Phys. 99, 08T102 (2006); 10.1063/1.2165928

Effect of heat treatment on microstructure and magnetic properties of anisotropic Nd-Fe-B films with Mo or $\mathrm{Ti}$ buffer layer

J. Appl. Phys. 98, 113905 (2005); 10.1063/1.2136208

\section{HIDEN}

Contact Hiden Analytical for further details: w www.HidenAnalytical.com E info@hiden.co.uk

\section{Instruments for Advanced Science}



Gas Analysis

dynamic measurement of reaction gas streams catalysis and thermal analysis

molecular beam studies

fermentation, environmental and ecological studies



Surface Science

UHVTPD

SIMS

end point detection in ion beam etch elemental imaging - surface mapping

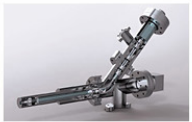

Plasma Diagnostics ' plasma source characterization etch and deposition process reaction kinetic studies analysis of neutral and radical species

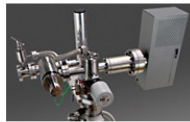

Vacuum Analysis partial pressure measurement and control of process gases reactive sputter process control vacuum diagnostics vacuum coating process monitoring 


\title{
Effect of Ta buffer layer and thickness on the structural and magnetic properties of Co thin films
}

\author{
K. Vahaplar, S. Tari, ${ }^{\text {a) }}$ H. Tokuc, and S. Okur \\ Department of Physics, Izmir Institute of Technology, Urla-Izmir 35430, Turkey
}

(Received 23 February 2009; accepted 12 July 2009; published 26 August 2009)

\begin{abstract}
Single $\mathrm{Co}$ and $\mathrm{Ta} / \mathrm{Co}$ bilayers were grown on $\mathrm{Si}(100)$ substrates in a magnetron sputtering system. The effect of Ta buffer layer and the thickness of Co layer on the structural and magnetic properties of the Co layers has been studied. A single Co layer shows a textured structure above thickness of $40 \mathrm{~nm}$ according to the $\mathrm{x}$-ray diffraction (XRD) pattern. The magnetic properties of Co layers depend significantly on the thickness of the films. Ta grows as highly textured $\beta$-Ta (tetragonal) phase on Si with a smooth surface. The XRD and atomic force microscopy results show that the Ta buffer layer improves the structural properties dramatically, resulting in a strongly textured and smoother surface morphology. The Ta layer also affects the magnetic properties of Co layers to a large extent, especially inducing an in-plane anisotropy in thin Co films. (C) 2009 American Vacuum Society. [DOI: 10.1116/1.3196784]
\end{abstract}

\section{INTRODUCTION}

Magnetism and nanostructured magnetic devices have attracted great research interest recently because of their high application potentials in magnetic sensors, spin valves, and magnetic storage industry. ${ }^{1} \mathrm{Co}$, with a high spin polarization at the Fermi level, is one of the most important materials for applications. ${ }^{2}$ It is also used in microelectronic devices due to its low electrical resistivity and small lattice mismatch with silicon. ${ }^{3}$ It is known that the magnetic properties, such as coercivity, remanence, and anisotropy of ferromagnetic materials, are strongly influenced by their surface roughness, ${ }^{4}$ film thickness, ${ }^{5}$ and crystallinity. ${ }^{6}$ It was also reported that magnetic properties are strongly affected by the deposition of an underlayer. ${ }^{7}$ Especially, the effect of a Ta seed layer on various thin ferromagnetic films has been studied. ${ }^{8-10}$ However, Ta and Co thin films have not been reported to the best of our knowledge. Understanding the growth, microstructure, and the effect of a buffer layer on the structure and magnetic properties of Co thin films will contribute to the field of thin film magnetism.

In this study, we report on the growth of $\mathrm{Si} / \mathrm{Co}$ and $\mathrm{Si} /$ $\mathrm{Ta} / \mathrm{Co}$ bilayer structures. The structural and magnetic properties of the Co films and the effect of the Ta buffer layer have been studied with various characterization techniques.

\section{EXPERIMENT}

$\mathrm{Co}$ and $\mathrm{Ta} / \mathrm{Co}$ bilayer films were grown on $\mathrm{Si}(100)$ substrates using a magnetron sputtering system with a base pressure of $1 \times 10^{-7}$ Torr equipped with a load lock, 2 in. magnetron guns, and a thickness monitor. rf substrate bias was applied for $3 \mathrm{~min}$ at $15 \mathrm{~W}$ to clean the substrate surface by etching in argon plasma before deposition of the films. This procedure removes about $\sim 30 \AA$ of the Si layers. Argon gas flow of 12 SCCM (SCCM denotes cubic centimeters per

\footnotetext{
${ }^{\text {a) }}$ Author to whom correspondence should be addressed; electronic mail: suleymantari@iyte.edu.tr
}

minute at STP) assured 3 mTorr of deposition pressure. Ta and Co targets with purity of $99.95 \%$ were used. The targets were presputtered prior to each layer deposition for $2 \mathrm{~min}$. The growth rate was $0.2 \AA / \mathrm{s}$ for Ta and $0.1 \AA / \mathrm{s}$ for Co. Various thicknesses of Ta and Co films, ranging from 4 to $100 \mathrm{~nm}$, as single and bilayers were grown by dc magnetron sputtering. All the layers were grown at ambient temperatures and in the same pump down cycle. No magnetic field is applied to the substrate during deposition.

The structural properties of the films were studied by x-ray diffraction (XRD) using $\mathrm{Cu} K \alpha$. The thickness of the layers was calibrated with a thickness monitor. The surface morphologies of the Co and Ta layers were examined with a Solver P47H atomic force microscope (NT-MTD) operating in tapping mode in air at room temperature. The magnetic hysteresis loops of single and bilayers were recorded by vibrating sample magnetometer (VSM) at room temperature.

\section{RESULTS}

Figure 1 shows the XRD pattern of the as-grown Co thin films as a function of thickness. There is no peak in the pattern representing Co up to a thickness of $\sim 40 \mathrm{~nm} .{ }^{11}$ Co starts to show a textured structure above $40 \mathrm{~nm}$ with a peak at $44.53^{\circ}$ representing hexagonal (002) planes. There is a small increase in the XRD signal intensity and in grain size as the thickness of the Co film increases. The average grain sizes for 50, 75, and $100 \mathrm{~nm}$ Co films were found to be 15.3, 17.4, and $20.4 \mathrm{~nm}$ from the Scherer formula, respectively. This indicates that the textured (crystalline) structure of Co improves slightly with the thickness. There is also a broad but less intense peak at $41.7^{\circ}$ coming from Co (100) planes for thicker films. There is no significant shift in the peak positions, indicating relaxed layers.

Co films were grown on the Ta buffer layer to investigate the effect of $\mathrm{Ta}$ on the structural and magnetic properties. Figures 2(a) and 2(b) show the XRD patterns of the $\mathrm{Si} / \mathrm{Ta}(7$ $\mathrm{nm}) / \mathrm{Co}(25 \mathrm{~nm})$ and $\mathrm{Si} / \mathrm{Ta}(40 \mathrm{~nm}) / \mathrm{Co}(25 \mathrm{~nm})$ bilayers, respectively. For $\mathrm{Ta}(7 \mathrm{~nm})$, there is no peak that represents $\mathrm{Ta}$; 


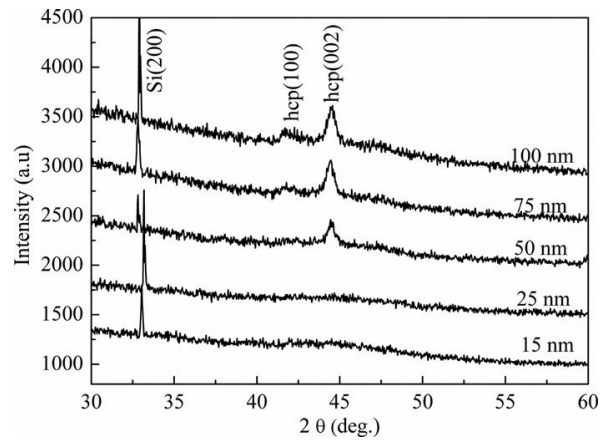

FIG. 1. XRD patterns of various thicknesses of Co thin films deposited on $\operatorname{Si}(100)$.

probably it is very thin or has not gained a textured phase yet at this thickness. However, Co shows a single intensive peak at $44.7^{\circ}$, indicating a strong crystalline structure which did not show a textured phase on bare Si. The peak at $34^{\circ}$ for $\mathrm{Ta}(40 \mathrm{~nm})$ represents tetragonal-tantalum ( $\beta$-Ta) (200) planes. It is well known that Ta has two structural phases: bcc cubic and $\beta$-Ta. ${ }^{12}$ The full width at half maximum of the Ta peak is $0.398^{\circ}$ and the corresponding grain size is $21 \mathrm{~nm}$. The thickness of the Ta does not affect the structural quality of the $25 \mathrm{~nm}$ Co layer significantly. Grain sizes of Co layers are found to be 15 and $13 \mathrm{~nm}$ for 7 and $40 \mathrm{~nm}$ of Ta, respectively.

We also deposited Ta films on Si to see whether there is a structural improvement in Ta films. As seen from Fig. 2(a), the $\mathrm{Ta}(7 \mathrm{~nm})$ does not show a peak in the XRD pattern. Figure 3 shows the XRD patterns of $\mathrm{Ta}(40 \mathrm{~nm})$ and $\mathrm{Ta}(80$ $\mathrm{nm})$. There is a large intensity increase in the Ta peak with increasing thickness. The grain sizes are calculated as 20 and $29 \mathrm{~nm}$ for 40 and $80 \mathrm{~nm}$ Ta, respectively. This large intensity increase is clearly due to the increase in the thickness of the Ta film. The grain size increase indicates that the Ta film's textured structure improves with thickness. On the other hand, there is another peak at $71.3^{\circ}$, representing $\beta$-Ta(004) planes for $80 \mathrm{~nm}$ Ta.

Surface morphologies of the single Co, Ta, and Ta/Co films on the Si substrate for various thicknesses were obtained over a scan area of $3 \times 3 \mu \mathrm{m}^{2}$ by atomic force microscopy (AFM). Figures 4(a) and 4(b) show the surface

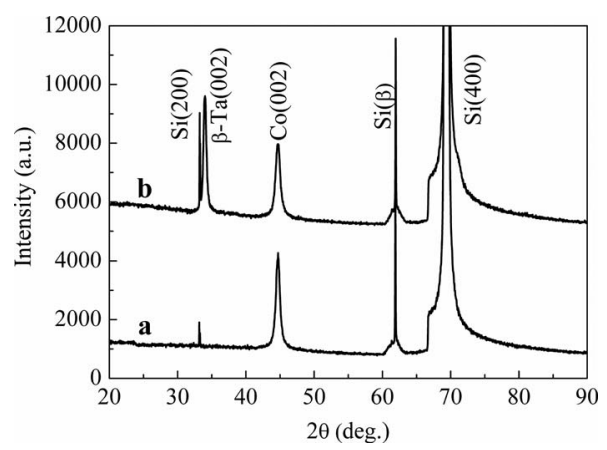

FIG. 2. XRD patterns of (a) $\mathrm{Si} / \mathrm{Ta}(7 \mathrm{~nm}) / \mathrm{Co}(25 \mathrm{~nm})$ and (b) $\mathrm{Si} / \mathrm{Ta}(40 \mathrm{~nm}) /$ $\mathrm{Co}(25 \mathrm{~nm})$ bilayers.



FIG. 3. XRD patterns of Ta films deposited on $\mathrm{Si}(100)$.

morphologies of the $\mathrm{Si} / \mathrm{Co}(25 \mathrm{~nm})$ and $\mathrm{Si} / \mathrm{Ta}(7 \mathrm{~nm}) / \mathrm{Co}(25$ $\mathrm{nm})$, respectively. The contrast shows a clear surface roughness difference between the two images. The rms roughnesses of $\mathrm{Si} / \mathrm{Co}(25 \mathrm{~nm})$ and $\mathrm{Si} / \mathrm{Ta}(7 \mathrm{~nm}) / \mathrm{Co}(25 \mathrm{~nm})$ are 5.0 and $1.3 \AA$, respectively. The Ta seed layer improves the structure of the Co film, resulting in a highly textured (see XRD in Fig. 2) and smoother surfaces. The AFM image of $\mathrm{Si} / \mathrm{Ta}(40 \mathrm{~nm}) / \mathrm{Co}(25 \mathrm{~nm})$ is shown in Fig. 5. As seen, there is a slight increase in the roughness of the Co film, $2.4 \AA$, as the Ta thickness increases when compared to $\mathrm{Si} / \mathrm{Ta}(7 \mathrm{~nm})$ / $\mathrm{Co}(25 \mathrm{~nm})$ [Fig. 4(b)]. This may be due to the slight increase in the roughness of $\mathrm{Ta}$ as its thickness increases.

The roughness of various thicknesses of the films is plotted in Fig. 6. For thin Co of 4 up to $15 \mathrm{~nm}$, roughness increases, followed by a decrease for thicker Co layers. There is no significant change in the roughness of the Ta and $\mathrm{Ta} / \mathrm{Co}$ layers. Overall rms roughnesses are between 2 and 6 $\AA$ for $\mathrm{Co}$ and between 1 and $2 \AA$ for the Ta and Ta/Co films. The initial increase in roughness in Si/Co films up to $15 \mathrm{~nm}$ maybe associated with the possible reaction between Co and $\mathrm{Si}$ at the interface, which might prevent the Co film from reaching its natural hexagonal structure for thin layers. The decrease in the roughness after $15 \mathrm{~nm}$ is possibly due to the fact that Co starts to gain its natural crystal structure. After thickness of about $50 \mathrm{~nm}$, the roughness of Co is almost constant. The roughness of the $25 \mathrm{~nm}$ Co film deposited on $\mathrm{Ta}(7 \mathrm{~nm})$ is much less than that of without the Ta buffer



(a)

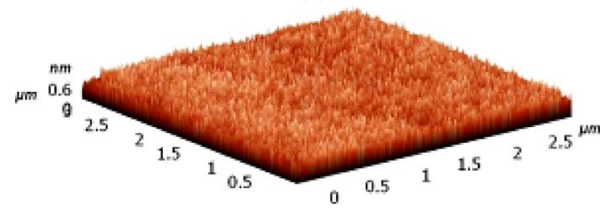

(b)

FIG. 4. (Color online) AFM images of (a) $\mathrm{Si} / \mathrm{Co}(25 \mathrm{~nm})$ and (b) $\mathrm{Si} / \mathrm{Ta}(7 \mathrm{~nm}) /$ $\mathrm{Co}(25 \mathrm{~nm})$. Roughnesses are 5 and $1.3 \AA$ for (a) and (b), respectively. 


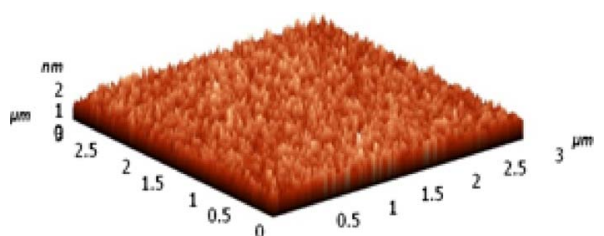

FIG. 5. (Color online) AFM image of $\mathrm{Si} / \mathrm{Ta}(40 \mathrm{~nm}) / \mathrm{Co}(25 \mathrm{~nm})$. Roughness is $2.4 \AA$.

layer. This is apparently due to the improvement of the textured feature of the Co layer when grown on Ta. It is also shown that the grain size of the $25 \mathrm{~nm}$ Co film on Ta is calculated as $15 \mathrm{~nm}$, whereas the same thickness of $\mathrm{Co}$ on $\mathrm{Si}$ does not show any peak in the XRD pattern. Although we do not see a peak for $7 \mathrm{~nm} \mathrm{Ta}$, Ta is already somewhat ordered and Co gains a textured phase on this ordered and smooth surface for much lower thicknesses. This may be associated with the better agreement between the structures of Co and Ta films compared to Co film and Si substrate. These Ta/Co surfaces are quite smooth for overlayer growths. In the case of rough surfaces, roughness may induce in-plane magnetic poles, which may result in coupling of magnetic layers for spin valve applications.

Magnetic hysteresis loops $(M-H)$ of all layers were recorded by VSM. Magnetic field is applied parallel to the film planes. The $M-H$ loops of the Co films of various thicknesses deposited on $\mathrm{Si}$ are shown in Fig. 7. There is not much change in the shape of the loops except for the 15 and $25 \mathrm{~nm}$ Co films for which the magnetization drops (sharp magnetization reversal) sharper than other thicknesses.

Figure 8 shows the plot of the coercive field $\left(H_{c}\right)$ and saturation magnetization $\left(M_{s}\right)$ versus Co thickness. The $H_{c}$ and $M_{s}$ depend strongly on the film thickness. ${ }^{13,14}$ The $H_{c}$ of the Co films decreases with increasing film thickness up to a threshold value, above thickness of $15 \mathrm{~nm}$. This observed low $H_{c}$ indicates the soft magnetic nature of thin Co films. The $H_{c}$ of $4 \mathrm{~nm}$ Co film is 72 Oe and it decreases sharply to 27 Oe for $15 \mathrm{~nm}$ thick Co films. This sharp decrease in $H_{c}$ may be associated with the formation of $\mathrm{CoO}$ on top of the Co film because the magnetic effects of antiferromagnetic



FIG. 6. Variation in roughnesses of $\mathrm{Co}$, Ta, and $\mathrm{Ta}(7 \mathrm{~nm}) / \mathrm{Co}$ as a function of thickness.



FIG. 7. Hysteresis loops of Co layers as a function of thickness deposited on Si.

layers are inversely proportional to the magnetic film thickness. The presence of the $\mathrm{CoO}$ on top of the Co films has no effect for thick layers. The effect of $\mathrm{CoO}$ on the properties of the Co thin film has been studied in literature. ${ }^{15}$ Another possible explanation is the presence of stress in thin Co layer. There is no significant change in the $H_{c}$ between 15 and 50 $\mathrm{nm}$. It is known that stress in thin films relaxes as the film thickness increases by especially introducing line defects in the film. Therefore the stress is not likely to be present at this range in the Co layers. Also the $\mathrm{CoO}$ effect diminishes for thick layers. Above thickness of $50 \mathrm{~nm}$, where Co has a textured structure, $H_{c}$ starts to increase linearly due to an increase in the grain size. Large grains cause large $H_{c}{ }^{16}$

The $M_{s}$ value of $4 \mathrm{~nm}$ of the Co film is small and increases with thickness of up to $15 \mathrm{~nm}$, which is close to the magnetization of bulk Co $\left(1422 \mathrm{emu} / \mathrm{cm}^{3}\right)$, then decreases gradually. The initial low $M_{s}$ of Co may be associated with the formation of dead layers due to the reaction between the $\mathrm{Co}$ and the Si at the very interface as well as the formation of $\mathrm{CoO}$ on top of $\mathrm{Co}$, in which both effects will decrease the thickness of the magnetic Co film. The increase in $M_{s}$ up to $15 \mathrm{~nm}$ is due to the fact that the thickness of magnetic Co layers increases. The decrease after about $15 \mathrm{~nm}$ is likely due to the structural changes in the Co films, which start to gain textured feature.

The angular dependence of the magnetization of the Co films has been measured between $0^{\circ}$ and $180^{\circ}$ in plane. The squareness $(S)$ is plotted and is shown in Fig. 9. $S$ is almost constant for thicknesses of $4,8,50$, and $75 \mathrm{~nm}$, indicating



FIG. 8. Variation in $H_{c}$ and $M_{s}$ for various thicknesses of Co layers deposited on $\mathrm{Si}$. 




FIG. 9. Magnetic anisotropy for various thicknesses of Co layers. Measurements were taken for $0^{\circ}-180^{\circ}$ azimuthal angle.

isotropic magnetic behavior. However, $S$ shows an oscillating behavior for 15 and $25 \mathrm{~nm}$ Co films, which represents the existence of an anisotropic magnetic behavior with a twofold symmetry. Since $M$ is measured between $0^{\circ}$ and $180^{\circ}$, we cannot see the complete symmetry. Similar behavior can be seen in the $\mathrm{Co} / \mathrm{Fe}$ bilayers. ${ }^{17}$ The Co films between 4 and 15 $\mathrm{nm}$ do not show significant magnetic anisotropy since defects and stresses present at these thicknesses in the films may cause domain wall pinning centers. It is also possible that the interface reaction of $\mathrm{Co}$ with $\mathrm{Si}$ and $\mathrm{CoO}$ on top of the Co surface contribute to this magnetic behavior. In the range of 15-25 $\mathrm{nm}$, the Co film does not have a good crystalline order; therefore, the anisotropy does not arise from the magnetocrystalline anisotropy. Anisotropy may be arising from the quenched-in stresses in the film during deposition. ${ }^{18}$ Therefore, the magnetization reversal process is dominated by the magnetization rotation as the azimuthal angle changes from $0^{\circ}$ to $180^{\circ}$. For the thicknesses above $50 \mathrm{~nm}$, no anisotropy is observed since large grains of the crystalline Co film have large magnetic anisotropies that maybe oriented in different directions within the grains. Then, there exist many corresponding easy axes within the film, resulting in an increase in $H_{c}$ and the disappearance of the magnetic anisotropy. ${ }^{19}$

In general, tantalum or similar metal films are used as seed or contact layers before the deposition of magnetic electrodes in spintronic device applications. Therefore, thin Ta layers were deposited before Co to investigate the effect of Ta underlayer and its effect on the magnetic properties of the Co layers. Figure 10 shows the $M-H$ loops of the 4 and 25 $\mathrm{nm}$ Co films deposited on $7 \mathrm{~nm}$ Ta seed layer. The $H_{c}$ of $\mathrm{Co}(4 \mathrm{~nm})$ is measured as 40 Oe and decreased to 20 Oe for $\operatorname{Co}(25 \mathrm{~nm})$. This may be due to the fact that $\operatorname{Co}(25 \mathrm{~nm})$ film posses better textured structure and smoother surface as seen from the XRD and AFM data. The $\mathrm{CoO}$ formation on top of the Co layer also contributes to this behavior as proposed for $\mathrm{Co}(4$ and $15 \mathrm{~nm})$ deposited on $\mathrm{Si}$. There is an increase in $M_{s}$ with an increase in the thickness. The squareness increases slightly with an increase in the thickness, which is due to the structural improvement of the Co layer. Magnetization reversal is sharper for better ordered structures.



FIG. 10. Hysteresis loops of Co layers deposited on the Ta $(7 \mathrm{~nm})$ buffer layer.

The comparison of the hysteresis loops of $\mathrm{Si} / \mathrm{Co}$ and $\mathrm{Si} /$ Ta/Co is shown in Fig. 11. The $H_{c}$ of Co decreases from 75 to $50 \mathrm{Oe}$ for $\mathrm{Co}$ on $\mathrm{Ta}$ and improves the squareness due to the obvious structural improvements in the Co layer when grown on Ta. The important point here is that $M_{s}$ of Co on Ta $\left(\sim 1000 \mathrm{emu} / \mathrm{cm}^{3}\right)$ is much larger than the $M_{s}$ of Co on $\mathrm{Si}$ $\left(\sim 600 \mathrm{emu} / \mathrm{cm}^{3}\right)$ for thickness of $4 \mathrm{~nm}$. This indicates the possibility of reaction at the $\mathrm{Si} / \mathrm{Co}$ interfaces. The effect of the $\mathrm{CoO}$ formation on top of the $\mathrm{Co}$ film, which is effective for very thin layers, is the same for both layers. Therefore, it is not likely to contribute to the changes seen in $H_{c}$ and $M_{s}$. However, in Fig. 11(b), there is no change in $H_{c}$ but a relatively sharp fall in magnetization reversal. As shown in the XRD pattern (see Fig. 2), the $25 \mathrm{~nm}$ Co film grown on the Ta buffer layer has a highly textured structure. Magnetization reversal is dominated by domain wall motion. The thickness of the Ta underlayer does not change the magnetic properties of the $\operatorname{Co}(25 \mathrm{~nm})$ layers significantly. Both $H_{c}$ and $M_{s}$ are comparable and the measured value of $M_{s}\left(1250 \mathrm{emu} / \mathrm{cm}^{3}\right)$ is close to the bulk $M_{s}$ value of the Co films $\left(1422 \mathrm{emu} / \mathrm{cm}^{3}\right)$.

The squareness curves of $\mathrm{Co}$ and $\mathrm{Ta} / \mathrm{Co}$ layers have been compared to see the effect of the Ta layer. Figure 12 shows the $S$ for angles between $0^{\circ}$ and $180^{\circ}$. Although $\mathrm{Ta}(7 \mathrm{~nm})$ improves the structure of the $\operatorname{Co}(4 \mathrm{~nm})$, there is no significant change in the $S$ for the $\mathrm{Ta}(7 \mathrm{~nm}) / \mathrm{Co}(4 \mathrm{~nm})$. Somewhat ordered $\operatorname{Co}(25 \mathrm{~nm})$ and strongly textured $\mathrm{Ta}(7$ and $40 \mathrm{~nm}) /$



FIG. 11. Comparison of hysteresis loops of Co and Ta/Co layers. 




FIG. 12. Comparison of the magnetic anisotropies of $\mathrm{Co}$ and Ta/Co bilayers.

Co(25 nm) layers show similar behavior and both have strong in-plane anisotropy. This is associated with the stressmagnetostriction induced in highly ordered Co films for $\mathrm{Ta}(7$ and $40 \mathrm{~nm}) / \mathrm{Co}(25 \mathrm{~nm})$. A remarkable change is seen for $\mathrm{Ta}(40 \mathrm{~nm}) / \mathrm{Co}(4 \mathrm{~nm})$ bilayers. $\mathrm{Co}(4 \mathrm{~nm})$, which does not show anisotropic behavior on a bare Si substrate, here shows a strong in-plane anisotropy. This may be associated with the more stress induced in thin Co $(4 \mathrm{~nm})$ film by the $\mathrm{Ta}(40 \mathrm{~nm})$, which has better crystalline structure than that of $\mathrm{Ta}(7 \mathrm{~nm})$.

\section{CONCLUSIONS}

Co films grown on $\mathrm{Si}$ substrates show a textured phase above thickness of $40 \mathrm{~nm}$ according to our XRD results. The deposition of the Ta buffer layer improves the structural quality of the Co layers dramatically. The $\mathrm{Ta}(7 \mathrm{~nm})$ buffer layer transforms the $25 \mathrm{~nm}$ of probably poorly textured Co into a highly textured phase. AFM micrographs show that the sputtered films result in a quite smooth Co and Ta films and the Ta buffer layer decreases the surface roughness of Co. The thickness of Co layers affects the magnetic properties, both $H_{c}$ and $M_{s}$, dramatically. Strong in-plane magnetic an- isotropy is observed for only 15 and $25 \mathrm{~nm}$ thick Co layers grown on Si. Moreover, a relatively $40 \mathrm{~nm}$ thick Ta buffer layer induces in-plane magnetic anisotropy in the $4 \mathrm{~nm}$ Co layer, whereas there is no anisotropy if grown on $\mathrm{Ta}(7 \mathrm{~nm})$ and on bare Si.

\section{ACKNOWLEDGMENT}

This work was supported by The Scientific and Technological Research Council of Turkey under Grant No. TBAG-105T109.

${ }^{1}$ S. Y. Chou, P. R. Krauss, and L. Kong, J. Appl. Phys. 79, 6101 (1996).

${ }^{2}$ S. S. P. Parkin, Phys. Rev. Lett. 71, 1641 (1993).

${ }^{3}$ T. Meyer and H. von Känel, Phys. Rev. Lett. 78, 3133 (1997).

${ }^{4}$ M. Li and G.-C. Wang, J. Appl. Phys. 83, 5313 (1998).

${ }^{5}$ A. Kharmouche, S.-M. Chérif, A. Bourzami, A. Layadi, and G. Schmermer, J. Phys. D: Appl. Phys. 37, 2583 (2004).

${ }^{6}$ A. Sharma, R. Brajpuria, S. Tripathi, and S. M. Chaudhari, J. Vac. Sci. Technol. A 24, 74 (2006).

${ }^{7}$ X. Bi, L. Gan, X. Ma, S. Gong, and H. Xu, J. Magn. Magn. Mater. 268, 321 (2004).

${ }^{8}$ H. Lefakis, M. Benaissa, P. Humbert, V. S. Speriosu, J. Werckmann, and B. A. Gurney, J. Magn. Magn. Mater. 154, 17 (1996).

${ }^{9}$ M. J. Hadley, R. Atkinson, and R. J. Pollard, J. Magn. Magn. Mater. 246, 347 (2002).

${ }^{10}$ R. Law, R. Sbiaa, T. Liew, and T. C. Chong, Appl. Phys. Lett. 91, 242504 (2007).

${ }^{11}$ A. Sharma, S. Tripathi, R. Brajpuria, T. Shripathi, and S. M. Chaudhari, J. Nanosci. Nanotechnol. 7, 2041 (2007).

${ }^{12}$ A. Jiang, T. A. Tayson, L. Axe, L. Glanczuk, M. Sosnowski, and P. Cote, Thin Solid Films 479, 166 (2005).

${ }^{13}$ M. L. Munford, L. Seligman, M. L. Sartorelli, E. Voltolini, L. F. O. Martins, W. Schwarzacher, and A. A. Pasa, J. Magn. Magn. Mater. 226230, 1613 (2001).

${ }^{14} \mathrm{M}$. Li and G. C. Wang, J. Magn. Magn. Mater. 217, 199 (2000).

${ }^{15}$ T. R. McGuire, T. S. Plaskett, and R. j. Gambio, IEEE Trans. Magn. 29, 2714 (1993).

${ }^{16}$ J. Islam, Y. Yamamoto, and H. Hori, J. Magn. Magn. Mater. 310, 2234 (2007).

${ }^{17}$ M. H. Park, Y. K. Hong, S. H. Gee, M. L. Mottern, and T. W. Jang, J. Appl. Phys. 91, 7218 (2002).

${ }^{18}$ P. Sharma and A. Gupta, J. Magn. Magn. Mater. 288, 347 (2005).

${ }^{19}$ H. Hoffman, J. Appl. Phys. 35, 1790 (1964). 